\title{
Les sacrifices faits aux ancêtres chez les Bangwa
}

Sacrifices made to ancestors among the Bangwa

\section{Charles-Henri Pradelles de Latour Dejean}

\section{OpenEdition}

Journals

Édition électronique

URL : http://journals.openedition.org/span/448

DOI : $10.4000 /$ span.448

ISSN : 2268-1558

\section{Éditeur}

École pratique des hautes études. Sciences humaines

\section{Édition imprimée}

Date de publication : 1 septembre 1979

Pagination : 131-138

ISSN : 0294-7080

\section{Référence électronique}

Charles-Henri Pradelles de Latour Dejean, «Les sacrifices faits aux ancêtres chez les Bangwa »,

Systèmes de pensée en Afrique noire [En ligne], 4 | 1979, mis en ligne le 04 juin 2013, consulté le 19 avril 2019. URL : http://journals.openedition.org/span/448 ; DOI : 10.4000/span.448 
L E S S A C R I F I C E S F A I T S A U X A C H E Z L E S B A N G W A par Chartes-Henri. Eradelles de Latoir Dejean

Bangwa est une des cent chefferies qui recouvrent les plateaux du centre-ouest au Cameroun. La population étant dense, les Bangwa he sont pas regroupës par unités lignagères, mais par families nucléaires établies, dans les différents quartiers, au grë des terres disponibles. Malgré l'éparpillemetıt des résidences et l'exode rural, la cohésion des groupes iignagers er leurs inter-relations ont été en grande partie maintenues par le culte des ancètres, assuré par les successeurs des fondateurs de lignage. En effet, quand un heritier regroupe, une fois par an, les membres de son groupe lignager, ils invoquent tous ensemble leurs ancêtres, au cours d'un rité spécial, afin de rester solidaires les uns des autres. Avant d'envoyer unie fille en mariage dans un autre lignage, un père la fait entrer toute nue dans la case réservée aux ancètres et il s'adresse a ces derniers en ces termes : "Pères, pères, êcuutez-moi, voilä vocre fille qui nous quitte pour rejoindre son mari, faites qu'ils se couchent à deux at qu'ils se relèvent trois ou quatre ; 11 faut qu'elle nous donne des petits-enfants." Puis il se retoume vers elle er, tout en faisant ure trace de cerre sur sa poitrine en signe de bénédiction, il lui dit : "Tu es dans l'état oú tu es venue au monde, ainsi tu repars. si tu te conduisais mal dans la fanille de ton mari, tu insulterais tes peres qui reposent ici." Les ancêtres soqt invoqués pour maintenir unis leurs descendants agnatiques et pour être témoins au mariage de leurs descendances. Ils se trouvent être ainsi garants des deux principales relations de parenté : la filiation et l'alliance. Les offrandes áacrificielles qui leur sont apportêes aux titres d'ancêtres du père (fíliation) et d'ancêtres 
du père de la mère (alliance) sont cependant identiques. Nous allons donc d'abord dëcrire ces sacrifices, et nous montrerons ensuite que leur sens ne relève pas tant de leurs modalités (types d'offrande et contexte) que de la place qu'ils occupent dans la structure parentale. Nous pourrons alors indiquer d'où provient leur efficacité.

Tout dēfunt devient ancêtre si, un an après sa mort, son successeur détache la tête du squelette, l'enduit d'huile de palme, la saupoudre de kaolin pour qu'elle lui soit favorable et l'enterre dans un grand canari. Autrefois, les canaris étaient ensevelis à mi-hauteur dans les cases d'habitation, les ancêtres masculins résidant chez leurs fils-héritiers, et les ancêtres féminins chez leurs filles-hêritières. Actuellement les croyances chrétiennes et occidentales ayant progressé, les canaris sont mis dans une grande case spéciale. Les crânes des pères sont gênéralement alignés contre le mur à droite de la porte d'entrée, et ceux des mères à gauche. La mobilité sociale s'étant accêlêrée, il arrive que le successeur d'un père fasse, à la demande de sa soeur absente, les sacrifices sur le crâne de sa mère. On dit en bangwa que 1'officiant "posent les champignons (ou la sauce) sur la tête des ancêtres", jü $m \alpha^{\prime}$ po ( $\left.n \alpha^{\prime}\right)$ ne tsuâ. Il n'y a pas de mot signifiant "sacrifier", ou rapprochant ce type d'offrande de la notion de sacré. Selon la logique bamiléké, les ancêtres ne sont pas demandeurs de sacrifices réguliers. Ni exigeants, ni jaloux comme les dieux occidentaux, ce sont des divinités domestiques qui vivent paisiblement et quotidiennement à côtê des humains. Hormis les deux cas que nous avons sus-mentionnés, elles ne sont invoquées qu'en cas d'infortune.

Un malade arrivant de la ville dit en français pour expliquer son dêplacement : "Je viens voir ma famille pour faire la coutume", et en bangwa : me nggiè tsiè ndu, "je viens lever la malédiction". Le nău signifie la malédiction et le nggandu celui qui souffre du mal envoyé par les ancêtres. Aussi la question que se pose l'infortuné est : "Je me demande ce que les ancêtres me veulent ?" I1 consulte alors le devin. On dit en bangwa que 1 'oracle cherche (mbi tchek), mais cela ne veut pas dire qu'il cherche par lui-même (mbi shuo); il cherche en s'adressant à un autre et plus précisêment à l'araignêe mygale. Cet animal apporte des éléments de réponse que le devin interprète et traduit en termes de nomination. "C'est le crâne d'un tel, dit-il, qui te demande de Iui apporter une chèvre, un coq..." Le malade doit remettre un bouc, 
un coq, du gibier ou des champignons cuits à l'eau aux ancêtres masculins, et une petite holle en fer, une poule, le foie ou la patte d'une chèvre cuite avec du plantin aux ancêtres fêminins. En principe l'animal doit être du même sexe que 1 'ancêtre auquel on 1 'offre, mais cette règle n'est pas toujours appliquée. J'ai souvent vu des chèvres offertes à la place de boucs. Par contre, on ne donne jamais de petites houes en fer aux hommes et de gibier aux femmes à 1'exception des mères de chef. Les morts sont ainsi sexueliement différenciés comme ils l'étaient de leur vivant.

Une autre règle plus stricte veut que la viande crue soit réservée aux mauvais morts (1), dont les canaris restent dehors sous l'auvent de la grande case, et que la viande cuite soit donnée aux bons morts, dont les canaris sont placés à 1'intérieur. Le coq, destiné aux premiers, est déchiré et abandonné sur les canaris et personne n'a le droit d'y toucher, tandis que le coq ou la viande de chèvre, destinés aux seconds, sont grillés à la flamme (mbi tuo) à l'intérieur de la grande case et 1 'héritier a le droit d'en manger. Remarquons que ce mode de cuisson est uniquement réservê à la nourxiture des ancêtres. Les humains se nourrissent d'aliments boulllis à l'intérieur des cases, à I'exception de ceux qui, soumis à un interdit (2) mangent leur nourriture grillée à la cendre (mbi fop). Dans l'endocuisine et l'exocuisine, les modes de cuisson différencient la nourriture des vivants et des morts.

\section{Lieux de aisson inteme exteme}

Destinataires

Les humains bouilii grillé à la cendre

Les ancêtres grillé à la flamme cru

En sacrifiant, 1'hêritier dit : "Pères, pères, levez-vous, voici votre enfant qui vous apporte quelque chose. Il est venu de loin, il

1. Les mauvais morts sont des hommes morts soit avec un gros ventre plein d'ascite, soit avec des jambes gonflëes, ou encore des personnes mortes par accident.

2. Les conjoints d'un mauvais mort et les tueurs de panthère doivent manger du plantin grillé pendant un certain temps. 
est venu pour vous. Ecoutez son appel, recevez ses souffrances, venez près de lui, luttez sur sa tête. Ecoutez le commerçant qui a de bons objets, les vend bien et cher. Ouvrez lui les yeux pour qu'il devienne riche." Ensuite, il bénit le malade venu apporter les offrandes en faisant sur sa poitrine un trait avec de la terre prélevée près des crânes. Le ndu est alors levé.

A quels ancêtres sont offerts ces sacrifices ? Une petite enquête rêalisée auprès de quarante-quatre héritiers indique que les enfants de fille apportent à eux seuls les deux-tiers des offrandes aux ancêtres du père de leur mère (cf. tableau 1). Ces résultats confirment ce que les Bangwa disent au sujet du ndu, à savoir que celui des ancêtres du grand-père maternel est plus fort que celui des ancêtres du père.

Membres de la famizle venant sacrifier sur ta tête d'un père Tableau 1

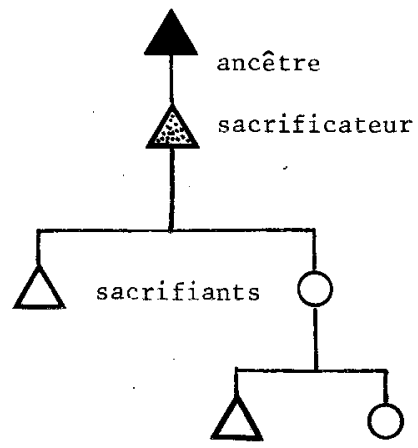

\begin{tabular}{|c|c|c|}
\hline \multicolumn{3}{|c|}{ grand-père mort } \\
\hline \multicolumn{3}{|c|}{ père } \\
\hline fils & \multicolumn{2}{|c|}{ filles } \\
\hline 4 & \multicolumn{2}{|c|}{10} \\
\hline \multirow[b]{2}{*}{ TOTAL : 68} & $\begin{array}{l}\text { petits- } \\
\text { fils }\end{array}$ & $\begin{array}{l}\text { petites } \\
\text { filles }\end{array}$ \\
\hline & 45 & 9 \\
\hline
\end{tabular}

L'enquête menée auprès des héritières des ancêtres féminins présente des résultats moins significatifs car la plupart des femmes devenues chrétiennes ne font plus de sacrifices. D'après les anciennes, les ancêtres féminins étaient autrefois nourris par les filles des filles des filles de leurs filles. Les femmes sacrifiaient surtout sur "la tête de leur mère troisième", c'est-à-dire une ascendante utérine du lignage dont elles ne relevaient pas. En effet, celles qui sont reliées entre elles par trois génërations et trois lignages appartiennent au même matri-groupe, elles n'ont plus de liens filiaux avec la quatrième gênération ascendante (cf. schêma 1). Les ancêtres privilégiés d'un homme et d'une femme ne sont donc pas des ancêcres par filiation, 
mais des ancêtres par alliance. Ce que nous ailons expliquer en comparant d'abord les attitudes qu'un individu entretient avec son père et le père de sa mère.

Scréma 3

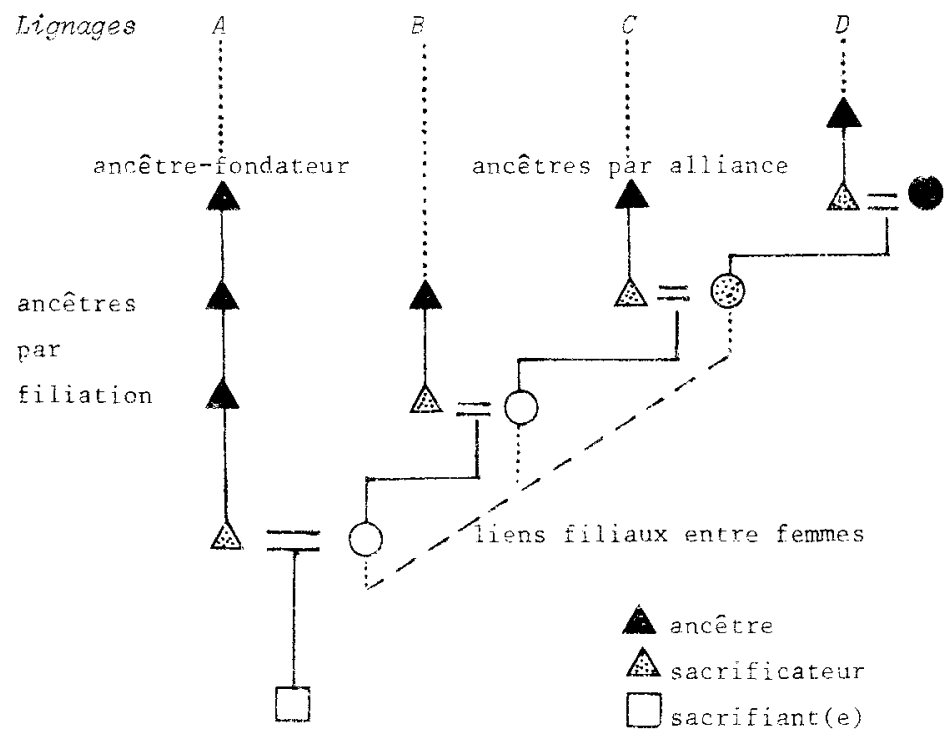

Un père est trè̀s proche de son fils. Il lui donne le nócessaire, le nourrit, l'habille, lui paye son ecolage et lui offre, s'il le peut, une terre et une femine, pour s'installer. Il actend en concrepartia qu'il subvienne a ses besons dans ses vienx jours. "La vieille feme, dit-on, tête les seins de safille." Le fils n'est pas pour autant contraint d"aider ses parents. Tout dépend de sa bonne volonté. Si le père donne beaucoup et atcenc autant en recour, le père de la mère, par contre, ne donne rien et n'attend rien du fils de sefille. Il ne lui offre ni terre, ni argent, ni tenme et, s'il lui fait un prêt, il doit être rembourse rapidetient pour ne pas devenir mâk mik, cause de malédiction. Le naiu est alors envoye par les ancêrres du grand-père maternel, il faut lui apporter des ottrandes pour les apaiser.

Le rôle du père est aussi de conseiller son tils, de iui apprendre 
ce qu'il faut faire et ne pas faire, de lui "montrer le chemin", pour qu'il suive ses traces. Souvent père et fils s'entraident et se font des confidences. Mais si le fils ne répond pas à l'attente paternelle, "s'il détourne", comme on dit en bangwa, le père peut le maudire en versant de l'eau dans un trou réservé à cet effet et situé dans sa concession. Leurs rapports sont alors irrémédiablement rompus, le fils ne peut plus retourner sur la terre de son père. La relation qui les unit est inverse de celle qui existe entre un grand-père maternel et son petit-fils. Ces derniers se parlent peu et ne se confient pas 1'un à 1'autre. "Tout ce que je dis à mon petit-fils, m'a déclaré un Bangwa, je pourrais aussi bien le dire à n'importe qui." Bref, ils ne sont pas intimes mais ils ne se querellent pas, et le grand-père maternel ne peut, en aucun cas, maudire son petit-fils. Leur relation est ainsi fondée sur une confiance mutuelle qui n'est $\mathrm{j}$ amais mise en défaut. C'est pour cette raison que le corps des bourreaux de 1 a chefferie était constitué autrefois par les fils des filles du chef, car ce dernier leur faisait plus confiance qu'à ses serviteurs pour les exécutions capitales. Enfin, lors des palabres, lorsque la défense d'un home était assurée par les nembres de sa famille, le chef qui jouait le rôle de juge était plus sensible aux arguments du grand-père maternel qu'à ceux du père, ou encore : nchu $\vec{a}$ tè njip ntchue ji tè, "1a parole du père de la mère est plus forte que celle du père".

La relation père/fils, souvent très étroite, peut évoluer de façon dramatique, tandis que la relation père de la mère/fils de fille, marquée par une certaine distance, s'avère en fin de compte plus sûre. Ces deux types de relation servent de support et de modèle aux rapports qu'un individu a avec les ancêtres de son père et ceux de son grandpère materne1.

Un chef de lignage porte généralement le "grand-nom" qui fut attribué au fondateur de sa lignée, aussi peut-il, par le truchement de 1 'homonymie, se substituer à lui et parler en son nom. "Je suis venu de tel et tel endroit", dit le chef Bangwa s'identifiant au premier chasseur de la région dont parlent tous les récits d'origine. L'héritier perpétue ainsi l'existence de 1 'ancêtre fondateur, ce qui lui confère 1 'autorité nécessaire pour regrouper ses descendants et les maintenir unis. Par contre, le même chef de lignage ne peut pas parler au nom de son ancêtre fondateur pour justifier son autorité auprès des fils de 
ses filles, car son "grand-nom" est destinè à s'effacer à travers 1a chaîne des alliances (cf. schéma 1). Ses descendants appartenant à la quatrième génération l'auront oublié. Les ancêtres du père de la mère ne sont pas, à l'instar de l'ancêtre-fondateur d'un lignage un terminus a quo immortel; ils sont mortels renvoyant à d'autres ancêtres sur $1^{\prime}$ axe des alliances. Ils ne sont pas non plus sacrés comme le montre la coutume appelée chi $2 a^{\prime}$. Lorsque quelqu'un laisse tomber par inadvertance de la nourriture, celle-ci revient de droit aux ancêtres de la concession. "Les ancêtres, dit-or, leur rendent les offrandes qui leur ont été apportées". Ils entretiennent ainsi une relation directe avec leurs descendants utérins, sans passer par 1 'intermédiaire du grand-père materne1. En d'autres termes, ils sont distincts de leurs héritiers. Ils n'ont pas besoin de se confondre avec eux. D'une façon générale, les ancêtres par alliance sont plus distants et plus sévères que les ancêtres paterne1s ; leur ndu, avons-nous déjà dit, est plus redouté. Bien que les offrandes sacrificielles remises à ces deux types d'ancêtre soient identiques, il est maintenant clair qu'elles n'ont pas la même signification, car un Bangwa entretient avec chacun d'eux, à travers leurs représentants respectifs, des rapports fondamentalement différents.

En effet, lorsqu'un homme apporte des offrandes sacrificielles à ses ancêtres paternels, son père, sacrificateur, est plus qu'un simple médiateur, puisqu'il peut se substituer à $1^{\prime}$ ancêtre fondateur. Il participe à son immortalité et appartient ainsi à la sphère du sacré. L'opération sacrificielle peut être représentẻe par le schéma suivant :

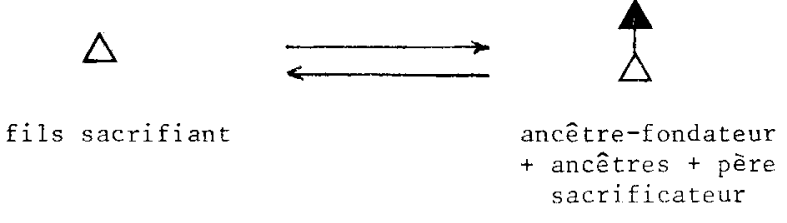

Le sacrifice fait aux ancêtres du père de la mère ne suit pas la même logique parce que le sacrificateur est distinct de ses ancêtres. L'opération sacrificielle comprend trois actants, le fils de la fille, 
le père de la mère et les mères de ce dernier. Le sacrificateur renvoie ainsi le sacrifiant à des ancêtres mortels et n'appartenant pas à la sphère du sacré.

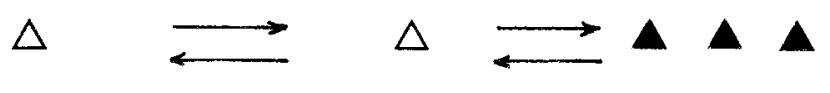
fils de la fille père de la mère pères du père de la mère sacrifiant sacrificateur ancêtres

Or si les Bangwa ont le plus souvent recours à ce deuxième type d'offrande, c'est que, pour eux, le sacrifice ne tient pas son efficacité de la participation au sacré.

D'où tient-il alors son efficacité ? Le ndu n'ëtant ni sacré, ni une force transmissible, i. n'y a aucune passation de pouvoir dans ce type de sacrifice. Selon les termes mêmes des Bangwa, le ndu est une malédiction ; c'est un mal-dit qui peut être effacé, si les relations de parenté dont les ancêtres sont garants sont rëtablies dans leur bien-dire. En apportant plus particulièrement des offrandes aux ancêtres du père de la mère, un indiviau réitère symboliquement le contrat d'alliance que le lignage de son père a scellé avec celui de sa mère. Ce faisant, il l'accrédite et s'y réinscrit dedans. La relation sacrificielle ne tient donc pas ici son efficacité d'elle-même, mais de 1 'ordre auquel elle renvoie. C'est ce demier qu'il faudrait mieux connaître pour en savoir plus sur ce type de sacrifice fait aux ancêtres. 